cornmon static deformity of flat or pronated foot-a subject with which I propose to deal more at length in a subsequent article-that is to say, to pronation at the mid-tarsal joint, flattening of the longitudinal arch, and sometimes flattening of the transverse arch associated with pain in the metatarso-phalangeal joints. Callus exudation added to plantar malposition results in a very crippled foot. In all gunshot injuries of tarsus and metatarsus the surgeon should take care during the later stages of healing not to bandage the sole rigidly against a flat foot-piece, for if that be done every irregularity of bone will conduce to

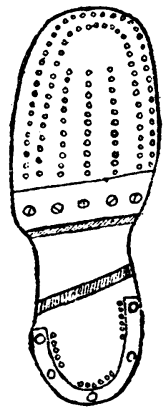

Frc. 12.-Boot with bar behind head to relieve injured metasure.

callosity when walking is resumed. It is necessary, therefore, at this stage to adjust a splint having an inside arch padded to conform to the natural shape of the foot Eversion of the foot should be guarded against, and the hollow of the arch should, when possible, be emphasized. Later, the heel of the boot should be raised on the inner side to obviate eversion, and, if the metatarsals are involved, in order to allow of early walking, a bar should be placed across the sole of the boot behind the tread (Figs. 12, 13). Light duty can then be undertaken at a much earlier date.

\section{Flail Joints.}

In answer to the question "What should be done with flail joints?" I would say, "Secure by operation an anky. losis in the most useful position." The only exception is in the case of the hip-joint where by means of simple mechanism a very useful limb may be obtained in spite of the joint being flail.

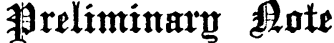

ON

\section{A NIETHOD FOR THE PREPARATION OF A NON-TOXIC DYSENTERY VACCINE.} BY

\author{
H. R. DEAN, M.D., F.R.C.P.,
} AND

R. S. ADAMSON, M.A., B.Sc.

(From the Pathological Laboratory of the University of Manchester.) (Report to the Medical Research Committee.)

The susceptibility of the rabbit to $B$. dysenteriae (Shiga) is well known, and constitutes a practical difficulty in the preparation of agglutinating serums for use in the recogni. tion of this micro-organism. During attempts to produce antiserum we ascertained that the intravenous injection of one-twentieth of a loop* of the culture of the particular strain which we were using, killed by heating for one hour at $58^{\circ} \mathrm{C}$., invariably produced the death of a rabbit in five to seven days. Smaller quantities frequently produced a

* Throughont this series of experiments we have expressed the quantities injected in loops and fractions of a loop. In all experiments we have used the same platinum loop, and cultures of the same strain, previously grown for twenty-four hours at $37^{\circ} \mathrm{C}$. If one loopful of the culture is emulsified in $1 \mathrm{c} \mathrm{cm}$. of saline solution and of this emulsion 1c.cm. is injected, the amount injected is reckoned as onefifth of a loop. The method is old-fashioned but provides comparable measurements, provided that the above precautions are adhered to. experiments contains from 160 to 180 million bacilli. fatal result. One strain of Shiga's bacillus was used in all our experiments.

The very marked success obtained by Lorrain Smith, Ritchie, and other's by the employment of eusol ${ }^{1}$ in the treatment of toxaemia and septicaemia suggested the possibility of its employment in vitro for the destruction of the toxins of the bacillary emulsion. 'The object of our experiments has been to modify the toxic properties of a bacillary emulsion of the Shiga bacillus without interfering with its capacity for producing immunity.

That bacillary antigens are not destroyed by hypo. chlorous acid had been shown by the successful use of antiformin extracts in the complement fixation reaction by Altmann and Schultz. ${ }^{2}$ In 1912 Dean $^{3}$ prepared a soluble typhoid antigen by precipitating an antiformin extract of typhoid bacilli with alcolhol. By this method a fine white powder was prepared, which was freely soluble in water. The solution so obtained was injected into a rabbit, and induced the formation of agglutinins against B. typhosus.

The results of our experiments on forty-seven rabbits have convinced us that it is possible by the use of dilute solutions of hypochlorous acid to prepare a Shiga vaccine, the injection of which is practically harmless to the rabbit, but is nevertheless followed by the production of a satisfactory degree of immunity.

The first few experiments showed that eusol very effectually destroys the Shiga toxin in vitro. The entire growth from three agar plates, which had been profusely inoculated. with the Shiga bacillus on the day before, was emulsified in $15 \mathrm{c.cm}$. of saline solution. To the emulsion was added an equal quantity of eusol, and the mixture was allowed to stand at room temperature for twenty-four hours. Of this mixture quantities containing 1 I $10^{1} \sigma 0$ of the entire growth on an agar plate were injected into the ear veins of three rabbits. No symptoms of any lind were observed, and all these rabbits remained in excellent health during the next seven days, when they received further inoculations. On the seventh day a sample of blood was taken, and agglutination tests were carried out. No formation of agglutinins could be demonstrated.

What is the smallest quantity of eusol which, when allowed to act on an emulsion of Shiga bacilli, will render the mixture non-toxic for rabbits?

In the endeavour to supply the answer to this question we have carried out experiments on twenty-four rabbits. The method employed throughout the series was the same. An emulsion was prepared by rubbing up one or more loops of a twenty-four hours' agar culture in a measured volume of saline. In the later experiments of the series the bacilli were killed by heating the emulsion for one hour at $58^{\circ} \mathrm{C}$. In the earlier experiments we relied on the bactericidal action of the eusol. The emulsion was mixed with an equal volume of diluted eusol and left for twentyfour hours at room temperature. The amount of the mixture that was injected was usually 1 c.cm. If, for example, it was decided to inject one-fifth of a loop of culture in a 1 in 1,000 solution of eusol, an emulsion of bacilli was prepared which contained two-fifths of a loop in 1 c.cm., and added to an equal volume of 1 in 500 eusol. The following examples are fairly typical of the results obtained:

\section{Rabbit 27.}

This animal was one of the three mentioned above. It had been inoculated on February 26th with one-hundredth part of the entire growth of an agar plate previously digested with an equal volume of eusol. On March 6th it was given an intravenous injection of Shiga emulsion, half a loop, in eusol 1 in 1,000. (The mixture was shown to be sterile.) The anima. remained perfectly well, and on March 10th Shiga emulsion, half a loop, in eusol, 1 in 1,000, was injected. This mixtur was found to contain living bacilli, for a few colonies of B. dysenteriae developed on an agar slope which had been inoculated with several yoops of the mixture. We liad been using throughout the experiment the same solution of eusol which had been prepared on February $25 \mathrm{th}$. It will be seen which had been prepared on February $25 \mathrm{th}$. It will be seen that a in 1,000 dilution of this solution sterilized the emulsion March 6th, but was unable to do so completely four day ater. This result was probably due to the deterioration of the eusol solution. If we had titrated our eusol solution before use and determined the hypochlorous acid content, we should probably have obtained more constant results. The result was, however, of interest, for the rabbit survived the inosulation with the living organism, and had evidently developed a high degree of immunity as a result of its previous inoculations with eusol-treated Shiga emulsion. The rabbit, in fact, showed no 
signs of illness after this inoculation. On March 17th a sample of its serum agglutinated a Shiga emulsion in dilution of 1 in 200.

On March 24th the animal was given an intravenous injection of Shiga emulsion, half a loop, previously killed by heat at $58^{\circ} \mathrm{C}$. but without the addition of any eusol: This represents at least ten minimal lethal doses, and a normal rabbit would almost certainly have died within forty-eight hours. The animal was quite well until March $27 \mathrm{th}$, when very slight signs of illness were observed. It was quite well on the following day. On April 3rd the agglutination titre of the serum was found to be 1 in 100. Marked complement fixation was observed in a dilution of 1 in 160 . On April 10th it was given an intravenous inoculation of two loops of heat-killed Shiga without eusol. No signs of illness developed, and the animal was quite weil te days later.

The emulsion which was injected into rabbit 27 on March 10th, and which was proved to contain living Shiga bacilli was injected on the same day in to three other rabbits, all of which had previously received two inoculations of a mixture of whigh emulsion and diluted eusol. Two of these animals sur. Shiga emulsion and
vived and one died.

Thus, in three cases out of four, rabbits immunized with a mixture of Shiga emulsion and eusol survived the injection of the living micro-organism. The injection of a living culture at so early a stage in the immunization of these animals was unintentional, but afforded evidence of the efficacy of the eusol-treated emulsions for the production of immunity.

We decided, however, to use in subsequent experiments emulsions of dead bacilli, and with this object all the emalsions used in subsequent experiments were heated for one hour at $58^{\circ} \mathrm{C}$. before the eusol was added. Cultures were made immediately before the eusol was added, and the emulsions were in every case found ta be sterile. The mixture of killed bacillary emulsion and diluted eusol was allowed to stand for twenty-four hours at room temperature.

It is generally held that the pathogenic action of the Shige bacillus is due to the presence of an endotoxin, and it is certain that heat-killed cultures of this microorganism are very virulent for rabbits. We decided, therefore, to determine the action of hypochlorous acid on the toxin of dead cultures. As the result of several experiments, we determined that eusol, in a dilution of 1 in 1,000 , greatly reduced, if it did not actually destroy the toxic action of heat-killed emulsions of Shiga bacillus Our next step was to determine the minimal quantity of eusol which would abolish the toxic action of a given quantity of emulsion of killed bacilli. The result of our experiment is shown in Table I. The dose of Shiga em. ployed was one-fifth of a loop $(160,000,000$ bacilli), a quantity which could be relied upon to kill a normal rabbit within a few days.

TABLE I.-Minimal Quantity of Eusol to Abolish Toxic Action. A.

\begin{tabular}{|c|c|c|c|c|c|}
\hline $\begin{array}{l}\text { No. of } \\
\text { Animal. }\end{array}$ & $\begin{array}{l}\text { Weight } \\
\text { in } \\
\text { Grams. }\end{array}$ & $\begin{array}{c}\text { Amount of } \\
\text { Killed } \\
\text { Shiga } \\
\text { Culture. }\end{array}$ & Eusol. & $\begin{array}{l}\text { Date of } \\
\text { Inocula- } \\
\text { tion. }\end{array}$ & Result. \\
\hline $\begin{array}{l}43 \text { (con- } \\
\text { trol) }\end{array}$ & 2,230 & $\begin{array}{l}\text { One-ffith } \\
\text { loov }\end{array}$ & 0 & March 15 & Died March 16. \\
\hline 42 & 2,130 & " & 1 in 16,000 & ", & Died March 25. \\
\hline 46 & 2,060 & " & 1 in 8.000 & " & Died March 16. \\
\hline 44 & 2,030 & $\bullet$ & 1 in 4,000 & - & Died March 16. \\
\hline 45 & 2,000 & $\bullet$ & 1 in 2,000 & " & Died March 19. \\
\hline 47 & 1,960 & n & 1 in 1,000 & " & $\begin{array}{c}\text { No sign of ill- } \\
\text { ness }\end{array}$ \\
\hline
\end{tabular}

B.

\begin{tabular}{|c|c|c|c|c|c|}
\hline 61 (con- & 2.210 & $\begin{array}{c}\text { One-fifth } \\
\text { loop }\end{array}$ & 0 & $\operatorname{March} 25$ & Died March 27. \\
\hline & 2,100 & " & 1 in 8000 & & Died March 31. \\
\hline 54 & 2,100 & " & 1 in 4.000 & " & Died March 27. \\
\hline 48 & 2,030 & " & 1 in 2,000 & " & \\
\hline 53 & 2,000 & " & 1 in 1,000 & 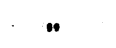 & No signs of ill- \\
\hline 50 & 1.750 & " & 1 in 500 & ״ & $\begin{array}{l}\text { No signs of ill- } \\
\text { ness. }\end{array}$ \\
\hline
\end{tabular}

It appears from the experiment shown in Täble $I, A$, that the toxin of one-fifth of a loop of culture of this Shiga strain was neutralized by the 1 in 1,000 , but not by the weaker dilutions of eusol which were employed. The survivor of the series, rabbit 47 , showed no signs of illness and re mained in perfect health. On April 4th we tested its immunity by the intravenous injection of two-fifths of a loop of a killed Shiga culture without any eusol. The animal remained perfectly well and had apparently developed \& satisfactory immunity as the result of one immunizing dose of Shiga treated with 1 in 1,000 eusol.

The result of a similar experiment is shown in Table I, I3: It entirely confirms the result slown in: Table $I, A$. The animal (No. 48), which was inoculated with 1 in 2,000 eusol in this case, survived after a transitory iliness which we noted on the second day after inoculation.

On April 10th the immunity of the three survivors was tested by the intravenous injection of one loop of killed culture-a very large dose. The test proved too severe, and all the animals died.

\section{On the Nature of the Immunity Produced by} Eusol-treated Emulsions of Shiga Bacilli.

The experiments above quoted and others, of which wo have notes, lead to the conclusion that it is quite possible to produce an effective and almost non-toxic vaccine by allowing a weak solution of eusol to act on a heat-killed emulsion of $B$. dysenteriae (Shiga). We have employed both intravenous and subcutaneous methods of injection, and, except in cases where we have intentionally given enormous doses, we have never observed any at all serious results. Intravenous inoculation commonly produces no sign of illness, and subcutaneous injections are followed at the most by a very slight degree of inflammation at the site of the injection. Rabbits after three, two, or exceptionally one inoculation with eusol-treated Shiga emulsion are able to sarvive the injection of a heated Shiga emulsion which contains many times the lethal dose for a normal unprotected rabbit. We have on many occasions tested the agglutinin content of the serum of the immunized rabbits, and on one occasion the antibody content of several serums was tested by the complement fixation method. The titre of the serum was in no case a high one, and, in the case of many animals, which were subsequently proved to be highly immune, there appeared to have been no production of agglutinins.

The result is not surprising in view of the fact that the agglutination titre of the serum from cases of Shiga dysentery is usually low as compared with the titre of serum from patients suffering from infections produced by typhoid and paratyphoid bacilli. It is moreover relatively difficult to obtain from a rabbit a really powerful antiShiga agglutinating serum by any method of immuniza. tion. In any case the presence or absence of agglutinins is, from the practical standpoint, of much less importance than the presence of active immunity, as demonstrated by a test injection of a large dose of virulent toxin.

The following experiments illustrate the method of immunization:

\section{Rabbit 34.}

On March 6th Shiga $\frac{1}{2}$ loop + eusol 1 in 1,000 subcutaneously.

On March 15th Shiga 1 loop + eusol 1 in 1,000 subcutaneously.

On March 25th Shiga 2 loops + eusol 1 in 500 subcutaneously.

No evidence of immunity could be obtained by agglutination and complement fixation tests.

On April 10th (test injection) two loops of heat-killed Shiga intravenously without eusol. This enormous dose produced no signs of illness, and the animal was alive and well on April 22nd.

Rabbits 39, 40, and 41 .

These three animals were immunized as follows :

On March 10th Shiga ${ }_{1}^{1}$ loop + eusol 1 in 100 subcutaneously.

On March 18th Shiga loop + eusol 1 in 500 subcutaneously.

On March 24 th Shiga loop + eusol 1 in 500 intravenously.

On April 4th 8 higa $\frac{1}{2}$ loop + eusol 1 in 1,000 intravenously.

The immunity was tested on April 10th by the intravenous. injection of one loop of heat-killed Shiga (without eusol) in the case of rabbits 39 and 41 . Rabbit 40 was given two loops of Shiga without eusol. No signs of illness developed, and all three animals were quite well on April 22nd.

We are inclined to think that the inoculations induce the formation of an antitoxin, the presence of which we are at present endeavouring to demonstrate in the serums of our immunized animals. 
The Action of Hydrogen Peroxide on Emulsions of the Shiga Bacillus.

We do not venture to express an opinion on the nature of the chemical reaction which takes place between the Shiga toxin and the hypochlorous acid. The result is that the bacillary emulsion loses its toxicity but not its capacity for acting as antigen. It is, of course, easy to say that toxin is converted into toxoid. But this is only another way of saying the same thing, and brings us no nearer to an explanation of what has happened. It is commonly believed that the poisonous properties of toxins are reduced by oxidation, and the possibility that hypochlorous acid destroyed the Shiga toxin by oxidation occurred to us. We have, in consequence, carried out a similar but limited series of experiments with hydrogen peroxide.

Raboit 37.

An emulsion of Shiga bacilli containing one loop of culture in $1 \mathrm{c.cm}$. was mixed without preliminary heating with an equal volume of $\mathrm{a} 1$ in 50 dilution of hydrogen peroxide in saline solution. After twenty-four hours 1 c.cm. of the mixture was injected subcutaneously. Cultures made from this mixture showed that it contained a few living Shiga bacilli. The animal died five days later.

\section{Rablit 36 .}

$\Delta \mathbf{n}$ emulsion of Shiga bacilli, containing one loop of culture in 1 c.cm., was mixed without preliminary heating with an equal volume of $a 1$ in 5 dilution of hydrogen peroxide in normal saline solution. The mixture was allowed to stand for twenty-four hours and then mixture was allowed to stand for mixture was injected beneath the skin of a rabbit on March 6th. On March 13th a sample of serum was obtained and produced slight agglutination of a Shiga emulsion in a dilution of 1 in 25 . On March 15th another subcutaneous injection was given (Shiga emulsion 1 loop, hydrogen peroxide 1 in 10). The shiga emulsion had been previously killed by heat, and was subsequently allowed to digest with the diluted hydrogen peroxide. On March 24th a Shiga emulsion was agglutinated by the serum in a dilution of 1 in 50 . On March 25th two loops of shiga emulsion (previously killed at $58^{\circ} \mathrm{C}$.) in 1 in 10 hydrogen peroxide solution were injected subcutaneously. The immunity of this animal was subsequently tested by the intravenous injection of two loops of killed Shiga emulsion. No signs of iilness were noticed and the animal remained perfectly well.

\section{Rabbit 38 .}

An emulsion containing one loop of Shiga culture (not killed by heat) was mixed with an equal quantity of undiluted hydrogen peroxide. After standing for twenty-four hours the mixture proved to be sterile, and $1 \mathrm{c.cm}$. was injected submixture proved to be sterile, and I c.cm. was injected subprobably destroyed not only the toxic but also the antigenic properties of the emulsion, for the second inoculation of one loop of heat-

\section{Rabbit 55.}

This animal was immunized by the subcutaneous inoculation of one-fifth of a loop of killed Shiga emulsion in 1 in 10 peroxid and later by a second dose of Shiga emulsion, half a loop i 1 in 10 peroxide. The immunity of this animal was tested by the intravenous injection of one loop of killed Shiga culture No signs of illness were noticed and the animal remained perfectly well.

The results obtained in this limited series indicate that hydrogen peroxide yields results of a similar character to those obtained by the employment of eusol.

\section{Experiments on Man.}

The results obtained from rabbits encouraged the hope that a harmless and effective prophylactic Shiga vaccine might be prepared on similar lines.

The vaccine was prepared by digesting a killed bacillary cmulsion of double the required strength with an equal volume of eusol (diluted 1 in 250 or 1 in 500) for twenty. four hours at room temperature. The injections were subcutaneous. Up to the present time three persons have been inoculated.

\section{CASE I.}

H. R. D. March 11th injected one-eighth of a loop killed Shiga in 1 in 1,000 eusol. Slight redness of skin developed around site of puncture after twenty-four hours. After fortyeight hours a circumscribed swelling developed in the subcutaneous tissue. The skin over the swelling became red, and firm pressure was slightly painful. The sivelling increased slightly up to the sixth day, then became smaller and disappeared about the tenth day. It was never very tender and the dis-
comfort produced was trivial; there were no constitutional symptoms.

The second dose, given on March 25th, consisted of half a loop of Shiga in 1 in 500 eusol; the local reaction was slightly more severe. On April 10th one-fifth of a loop ( 160 million bacilli) of killed culture without eusol was injected as a test of immunity. The local reaction was so slight that it could only be detected with difficulty.

\section{GASE II.}

R. S. A. On March 15th one-eighth of a loop killed Shiga in $1 \mathrm{in} 1,000$ eusol was injected, and on March 30 th one-fourth of loop killed Shiga in 1 in 500 eusol. There was no constitutional disturbance, and the local reactions were similar to those described in the first case.

\section{CASE IIr}

R. W. On March 16th one-eighth of a loop hilled Shiga in ensol 1 in 1,000 was injected, and on March 30th half a loop killed Shiga in eusol 1 in 500 . The local reaction after the first dose was a trifle more marked than in the other cases. The local reaction produced by the second dose was hardly appreciable On April 10th one-fifth of a loop (160 million bacilli) of killed culture without eusol was injected. There was practically no local reaction.

In all three cases constitutional symptoms were entirely absent. The local reaction was a little more marked than that which follows an injection of typhoid vaccine in an average case. It was usually well marked at the end of the second day, and reached its maximum on the fifth or sixth day. It caused none of us any appreciable in. convenience. Up to the present time we have not been able to demonstrate the presence of agglutinins in our serums.

Summary and Conclusions.

Our experiments up to this time show that:

1. The toxicity of an emulsion of $B$. dysenteriae (Shiga) can be enormously reduced, or even entirely destroyed, by the action of a dilute solution of eusol or of hydrogen peroxide.

2. Thie injection of such an emalsion in which the toxin has been destroyed is followed by the pro. duction of a satisfactory degree of immunity.

These results appear to us of sufficient interest to justify the publication of a preliminary note, but we are aware that there are several points which require further investigation.

The original object of these experiments was to diminish or destroy the toxicity of a Shiga emulsion without interfering with its immunizing properties.

The results have been obtained by rather rough methods. In subsequent experiments we propose to use mixtures of counted bacillary emulsions with solutions of eusol in which the content of hypochlorous acid has been deter. mined by titration. It may then be found possible to determine the time which the reaction requires at any given temperature. It will also be of interest to us to ascertain if it is possible to produce agglutinins against typhoid and paratyphoid bacilli by similar methods. It is possible that the method may be found useful in reducing the symptoms which follow the injection of $B$. typhosus and $B$. pestis vaccines.

With regard to the production of a suitable Shiga vaccine we suggest the following procedure :

Prepare an emulsion containing 200 million bacilli per cubic centimetre. Heat for an hour at $58^{\circ}$ or $60^{\circ} \mathrm{C}$. Mix with an equal quantity of a 1 in 500 solution of freshly prepared eusol in normal saline. Allow mixture to stand at room temperature for twenty-four hours. Inject sub. cutaneously $1 \mathrm{c.cm}$. of this mixture; $1 \mathrm{c.cm}$. will contain 100 million bacilli in a 1 in 1,000 dilution of eusol. The second dose might be given ten days later. Prepare an emulsion containing 800 million in 1 c.cm. Heat for one hour at $58^{\circ}$ or $60^{\circ} \mathrm{C}$. Mix with an equal quantity of 1 in 500 eusol. Allow to stand for twenty-four hours at room temperature and inject $1 \mathrm{c.cm}$. A third dose of 200 million or more bacilli, sterilized with heat but without the addition of eusol, may possibly be necessary to complete the production of a satisfactory immunity.

The above-suggestions are put forward tentatively as the result of a very limited number of experiments on man. It is quite likely that the dose might be considerably increased as the result of further experience. It is also possible that it may be found feasible to increase the relative amount of eusol in the mixture without diminish. ing its immunizing properties. Such an increase would be accompanied, in all probability, by a still further reduction in the local reaction.

Of the nature of the action of the eusol on the Shiga toxin wo are not in a position to offer an opinion. The 
fact that bydrogen peroxide appears to exert a similar action to eusol suggests that the reaction is of the nature of an oxidation process.

REFErEnces.
1 Lorrain Smith, Drennan, Rettie, and Campbell, Experimental Observations on the Antiseptic Action of Hypochlorous Acid and it Application 10. Treatment of cases of Tas October 9th, 1915; Lorrain Smith, Ritchie, and Rettie. On a Case of Septicaemia treated by Intravenous Injeclion of Eusol, BRITIsF Indical Journal. November 13th, 1915; Fraser ard Bates, The Treatment of Acule Toxaemia Secondary to Gas Gangrene by the Intravenous Injection of a Solution of Hypochlorous Acid, Britist MEDICAL JOURNAL, January 15th, 1916, p. 83 ; Clinical Report on the Applications of Eusol, Lalcet, 1916, February 5th and 2th. Althan and scherin Inmunten als Antigene bei der Soluble Typhoid Antigen. Proceedings of the Pathological Society of Great Iritain and Ireland. June, 1913, Journal of Pathology und Bacteriology, vol. xviii, p. 117 .

\section{THE THERAPEUTIC AND REACTION EFFECTS OF KHARSIVAN.}

\section{A RECORD OF 600 INJECTIONS.}

H. C. LUCEY, M.D., B.S.Lond.,

Temporart Captain, R.A.M.C., Rotal Herbert hospital, Wootwice.

When kharsivan, the British preparation of the same chemical constitution as salvarsan, was introduced last year, the Medical Research Committee made an appeal to members of the medical profession to furnish it with accurate records of the results, both as regards its therapentic efficacy and the occurrence of any special incidental symptoms.

For such records, in the treatment of syphilis, to be value, it is neceseary that (1) some definite scheme for recording results should be adopted, (2) all disturbing factors, such as errors in technique, should be eliminated.

A scheme such as that shown in the next column will be found useful.

It is convenient to keep a ledger embodying some such scheme as this, the details of which should be filled in every time a patient receives an injection.

In this way, besides noting the immediate reaction to a dose given on a particular date, a permanent record is kept of the patient's syphilitic history. The third case in the reaction scheme may be talien as an example. In the column "Previous 'I'reatment" it will be seen that the patient had an injection of kharsivan 0.6 gram on January 19th, with two mercurial injections to follow. On referring to the page in the ledger recording injections given on January 19th, details of his condition and reaction on that date will be noted, and it will be observed what progress he has made in the interval.

At the present time, as a routine for primary and secondary syphilis, I give three injections of kharsivan 0.6 gram at fortnightly intervals, with two weekly injections of mercurial cream in the intervals. This is a short intensive course, designed to render a soldier fit for active service as quickly as is possible consistent with a reasonable chance of a permanent cure.

That this course of treatment does gire him a reasonable chance is shown by Gibbard and Harrison's statistics produced at the International Congress of Medicine, 1913. On a precisely similar course, but using the German preparation " 606 ," they found that only $3.9 \mathrm{per}$ cent. of cases relapsed clinically and 19.4 per cent. serologically within a year of the suspension of treatment. It is, of course, not possible during ivar time to carry out systematic Wassermann tests or to examine patients at stated intervals for signs of relapse; but up to the present I have had only one man return to me, and that not for a relapse, but for a fresh infection.

I give this case with reserve as an example of re infection with syphilis, because, unfortunately, the diagnosis of primary syphilis in the first instance, though supported on clinical grounds, was not established beyond all doubt by the finding of Treponema pallidum. No cxamination of the exudate from the sore was made on the first occasion.

D. A. was admitted into hospital on August 12th, 1915, with a sore on the glans penis. This was typical of a syphilitio chancre in this situation, being of the papulo-erosive type,

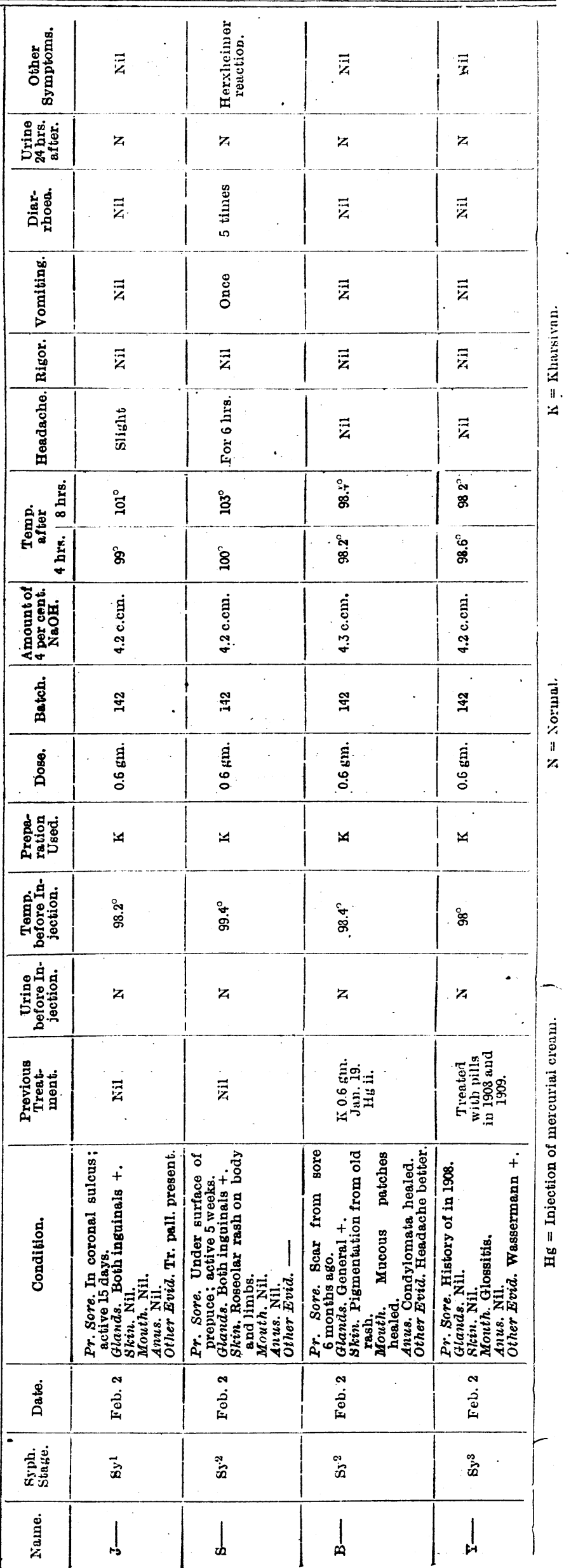

